\title{
Improved Solid-Phase Peptide Synthesis of Wild-Type and Phosphorylated Phospholamban Using a Pseudoproline Dipeptide
}

\author{
Shadi Abu-Baker*, Gary A. Lorigan \\ Department of Chemistry and Biochemistry, Miami University, Oxford, USA \\ Email: *abubaksi@muohio.edu
}

Received April 2, 2012; revised May 17, 2012; accepted June 13, 2012

\begin{abstract}
In this study, we report that the insertion of a pseudoproline dipeptide for the solid-phase peptide synthesis of wild-type Phospholamban protein (WT-PLB) has two important advantages. First, it disrupts the formation of different secondary structures, which is responsible for poor couplings during the preparation of highly aggregated sequences. Second, it enhances the purities and solubility of crude products leading to easier HPLC purification.
\end{abstract}

Keywords: Solid-State Peptide Synthesis; Pseudoproline Dipeptide; Phospholamban

\section{Introduction}

Phospholamban (PLB) is a hydrophobic 52-amino acid transmembrane protein that is involved in regulating the contraction and relaxation of heart muscle [1-3]. Phosphorylation of PLB by cyclic AMP- and calmodulindependent kinases is believed to increase the rate of calcium re-uptake by the sarcoplasmic reticulum and result in muscle relaxation [1-3]. The isolation and purification of large quantities of native PLB through molecular biology techniques has not yet been achieved due to difficulties encountered in the bacterial over expression of phospholamban cDNA [4,5]. Alternatively, PLB has been prepared by chemical synthesis using standard solidphase peptide synthesis and purification in organic solvents [6, 7]. In addition, this approach gives the opportunity to synthesize site-specific isotopically labeled peptides and proteins [8-10]. The biochemical and biophysical comparison of synthetic PLB and native PLB revealed that they are both similar in size and functionally identical $[6,7]$.

\section{Materials and Methods}

\subsection{WT-PLB Synthesis and Purification}

PLB was synthesized using modified Fmoc-based solidphase methods with an ABI 433A peptide synthesizer (Applied Biosystems, Foster city, CA). During our first run we found that the coupling of Leu-7 to Thr-8 was difficult even after double coupling and extending the

*Corresponding author. reaction time to six hours. However, this problem was solved by using the pseudoproline dipeptide of FmocLeu-Thr ( $\left.\Psi^{\mathrm{Me}, \mathrm{Me}} \mathrm{Pro}\right)-\mathrm{OH}$ from Novabiochem (San Diego, CA). The use of a pseudoproline dipeptide of FmocLeu-Thr ( $\Psi^{\mathrm{Me}, \mathrm{Me}}$ Pro)-OH enhanced the yield to about 25 $\%$ after lyophylization. To synthesize P-PLB, a pre-phos-phorylated Fmoc-serine amino acid was used at amino acid position 16 instead of the regular Fmoc-serine used in the synthesis of PLB. The crude peptide was purified on an Amersham Pharmacia Biotech AKTA explorer 10S HPLC controlled by Unicorn (version 3) system software. The purified protein was lyophilized and characterized by matrix-assisted laser desorption ionization time of flight (MALDI-TOF) mass spectrometry.

\section{Results}

\subsection{Solid-Phase Peptide Synthesis of WT-PLB}

The chemically synthesized form of the full length PLB (Figure 1(a)) and P-PLB (Figure 1(b)) was used for all of the solid-state NMR experiments. In general, solidphase peptide synthesis (SPPS) starts with the C-terminal amino acid attached to a solid support (resin). Amino acids are then coupled one at a time till the $\mathrm{N}$-terminus is reached. Each time an amino acid is added, the following three steps are repeated: First, deprotection of the Nterminal amino acid of the peptide bound to the resin (removal of the Fomc protecting group, see the aromatic part in Figure 2). This step is followed by activation and coupling of the next amino acid. And finally, the new N-terminal amino acid is deprotected [11]. 
H-Met1-Asp-Lys-Val-Gln-Tyr-Leu-Thr-Arg-Ser10-Ala-Ile-Arg-Ar g-Ala-Ser-Thr-Ile-Glu-Met20-Pro-Gln-Gln-Ala-Arg-Gln-Asn-LeuGln-Asn30-Leu-Phe-Ile-Asn-Phe-Cys-Leu-Ile-Leu-Ile40-Cys-LeuLeu-Leu-Ile-Cys-Ile-Ile-Val-Met50-Leu-Leu-OH

(a)

H-Met1-Asp-Lys-Val-Gln-Tyr-Leu-Thr-Arg-Ser10-Ala-Ile-Arg-Ar g-Ala-Ser $\left(\mathrm{PO}_{3} \mathrm{H}_{2}\right)$-Thr-Ile-Glu-Met20-Pro-Gln-Gln-Ala-Arg-GlnAsn-Leu-Gln-Asn30-Leu-Phe-Ile-Asn-Phe-Cys-Leu-Ile-Leu-Ile40Cys-Leu-Leu-Leu-Ile-Cys-Ile-Ile-Val-Met50-Leu-Leu-OH

(b)

Figure 1. Primary sequence of (a) PLB and (b) P-PLB. Sites of pseudoproline substitution are highlighted in red. P-Ser residue highlighted in blue was introduced using Fmoc-Ser (PO(OBzl)OH)-OH.

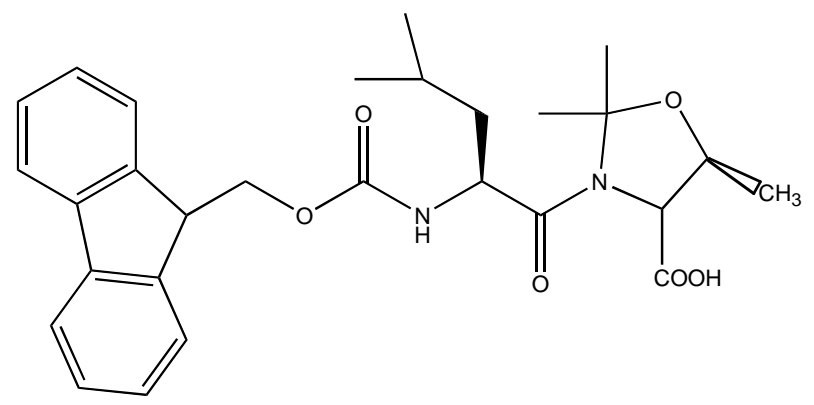

Figure 2. The pseudoproline dipeptide Fmoc-Leu-Thr(CMe, Mepro)-OH. This structure was generated using ChemDraw software and it is similar to the structure shown in the Novabiochem website [13].

To control the progress of the synthesis, the deprotection and coupling steps can be monitored using a UV detector. Several approaches including switching to different resins and activating reagents as well as using a pseudoproline dipeptide has been suggested to improve the yield of poor synthesis [12]. Figure 2 shows the pseudoproline dipeptide Fmoc-Leu-Thr(CMe,Mepro)-OH. In this dipeptide, the Thr residue has been reversibly protected as proline-like TFA-labile oxazolidine [13].

WT-PLB was synthesized according to a new procedure developed in the Lorigan's lab. Briefly, WT-PLB was synthesized using modified Fmoc-based solid-phase methods with an ABI 433A peptide synthesizer (Applied Biosystems, Foster city, CA). WT-PLB is very hydrophobic; thus, the synthesis of this peptide is very challenging. Nevertheless, by using a combination of extended coupling and deprotection protocols with a single pseudoproline dipeptide substitution, we were able to obtain both purified PLB and P-PLB in a yield of $25 \%$. Couplings were performed using 10-fold excess of Fmocamino acids activated with HBTU/DIPEA. The synthesizer was programmed to use conditional UV feedback monitoring; coupling and deprotection reactions are extended automatically, and a capping step introduced after the coupling step, based on the kinetic profile of the
Fmoc deprotection reaction. For certain residues additional extensions to the coupling times were used as indicated in Table 1.

All peptides were cleaved from the resin by treatment with TFA/EDT/thioanisole/water (10:0.5:0.25:0.5) for $2.5 \mathrm{~h}$, and isolated by centrifugation followed by precipitation with methyl $t$-butyl ether. PLB consists of a hydrophilic N-terminus (residues 1 - 20), a hinge region (21 - 30) and a hydrophobic $\alpha$-helical transmembrane tail (31 - 52) [1]. From previous work by Lorigan and co-workers [11], it is known that the synthesis of the C-terminal transmembrane region of PLB is extremely difficult, particularly the region from Cys36 to Cys45. To overcome these difficulties, the Lorigan group developed a strategy involving extended double coupling together with capping and conditional repetition of the Fmoc deprotection reaction [11]. Using this approach, PLB (24 - 52) segment was obtained in a purified yield of $37 \%$ [11].

Initially, we attempted the synthesis of full length PLB with standard amino-acid building blocks using the protocols previously described [11]. A PEG-PS resin (0.22 $\mathrm{mmol} / \mathrm{g}$ ) was selected as the solid support to reduce steric crowding and aggregation during chain assembly. Using the conditional feedback monitoring, this synthesis was completed in 9 days, as compared to 10 days for the shorter PLB (24 - 52) prepared on polystyrene resin [11].

UV monitoring of the Fmoc deprotection reactions indicated that the peptide assembly proceeded smoothly until Leu-7 (Figure 3(a)). However, following introduction of this residue, there was a marked decrease in the height of the Fmoc deprotection peak, indicating difficulties in the coupling of Leu-7 to Thr-8. Attempts to improve this coupling by double coupling or extending the reaction time to 6 hours had little effect. In view of the problems with the coupling of Leu-7 to Thr-8, the synthesis was repeated in exactly the same manner, except that Leu-7 and Thr-8 were introduced simultaneously using the pseudoproline dipeptide Fmoc-Leu-Thr(CMe, Mepro)-OH (Figure 2). In the presence of this dipeptide, UV monitoring of the Fmoc deprotection reactions indicated that the peptide assembly proceeded reasonably smoothly until the end of this synthesis (see Figure 3(b)).

Table 1. Coupling protocols used for assembly of PLB peptides.

\begin{tabular}{ll}
\hline \multicolumn{1}{c}{ Cycle } & \multicolumn{1}{c}{ Method } \\
\hline $2-5$ & Single coupling \\
$6,20-26,28,29$ & Single coupling +1 h extension \\
$7-14$ & Double coupling +6 h extension \\
$15-19,31,34-39$, & Double coupling \\
$41,42,47,49-51$ & \\
$27,30,33,40$, & Double coupling +2 h extension \\
$43-46,48$ & \\
\hline
\end{tabular}




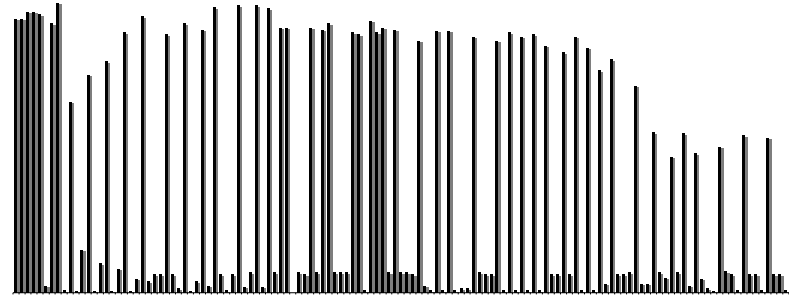

(a)

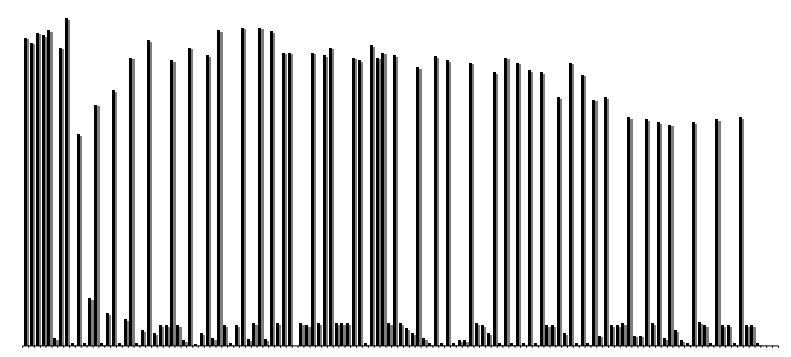

(b)

Figure 3. Traces from the UV monitoring of Fmoc removal during the synthesis of PLB using: (a) Standard amino acid building blocks; and (b) A pseudoproline dipeptide (Leu7Thr8).

\subsection{HPLC Purification of WT-PLB}

Following global deprotection and cleavage of the peptide from the resin, PLB was purified by preparative reverse phase chromatography (Figure 4) on a C4 column eluted with a gradient formed between $0.1 \%$ TFA in nanopure water (solvent A) and MeCN/isopropyl alcohol/water/TFA (38:57:5:0.1) (solvent B). After lyophilization and using standard Fmoc-amino acid building blocks (see Figure 4(A)), the purified peptide was obtained in a yield of only $9 \%$ based on initial resin substitution. Conversely, with the dipeptide (Figure 4 (B)), the purified PLB was obtained in a yield of $25 \%$, nearly a 3-fold increase when compared to the synthesis using standard building blocks.

\subsection{Characterization of WT-PLB Using MALDI-TOF}

When the dipeptide was used to synthesize WT-PLB, a correct mass of $6080 \mathrm{MU}$ was obtained after the purification step (Figure 5(A)). Conversely, when the dipeptide was not used, MALDI-TOF indicated the presence of an impurity with a mass of $5144 \mathrm{MU}$, which could be ascribed to Ac-PLB (9-52) (Figure 5(B)).

\section{Conclusion}

The insertion of a pseudoproline dipeptide improved the synthesis yield and purification of WT-PLB protein. This insertion has two important advantages. First, it disrupts the formation of different secondary structures, which is
(A) Without Dipeptide (PLB)

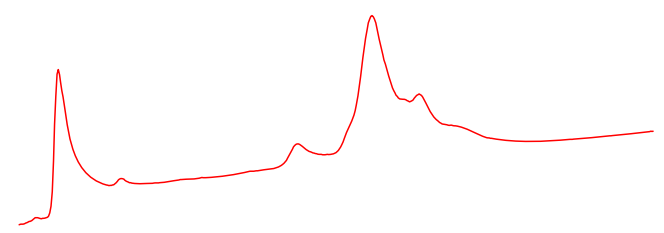

(B) With Dipeptide (PLB)
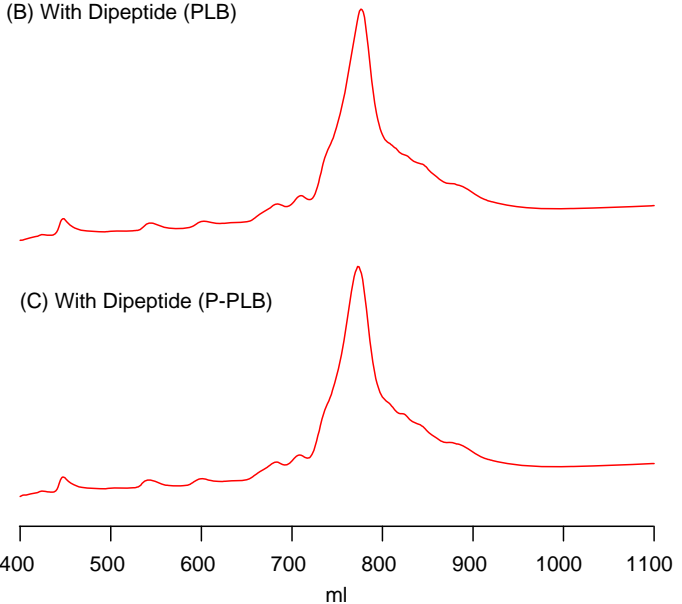

Figure 4. Preparative HPLC profiles of (A) WT-PLB prepared using standard Fmoc-amino acid building blocks; (B) WT-PLB prepared using a pseudoproline dipeptide; and (C) The phosphorylated form PLB (P-PLB, a pre-phosphorylated Fmoc-serine amino acid was used at amino acid position 16 instead of the regular Fmoc-serine used in the synthesis of PLB) prepared using pseudoproline dipeptide. HPLC conditions: C4 semi-preparative polymer-based column (259VHP82215, $8 \mathrm{~mm} 300 \AA \AA, 22 \mathrm{~mm} \times 150 \mathrm{~mm})$; buffer $A, 0.1 \%$ TFA in water; buffer $B$, MeCN/isopropyl alcohol/water/TFA 38:57:5:0.1; gradient, $5 \% \mathrm{~B}$ to $60 \% \mathrm{~B}$ in $25 \mathrm{~min}$ then $60 \%$ to $100 \%$ in $60 \mathrm{~min}$; flow rate, $10 \mathrm{ml} / \mathrm{min}$.

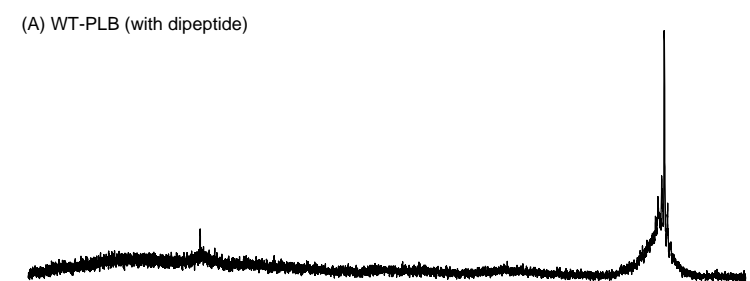

(B) WT-PLB (without dipeptide)

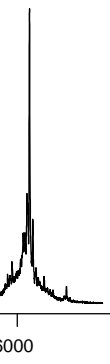

Figure 5. MALDI-TOF spectra of (A) WT-PLB prepared using a pseudoproline dipeptide; (B) WT-PLB prepared using standard Fmoc-amino acid building blocks. 
responsible for poor couplings during the preparation of highly aggregated sequences. Second, it enhances the purities and solubility of crude products leading to easier HPLC purification. This technique can be used for similar proteins that show poor synthesis.

\section{REFERENCES}

[1] H. K. B. Simmerman and L. R. Jones, "Phospholamban: Protein Structure, Mechanism of Action, and Role in Cardiac Function,” Physiological Reviews, Vol. 78, No. 4, 1998, pp. 921-947.

[2] P. James, M. Inui, M. Tada, M. Chiesi and E. Carafoli, "Nature and Site of Phospholamban Regulation of the Calcium Pump of Sarcoplasmic Reticulum," Nature, Vol. 342, 1989, pp. 90-92. doi:10.1038/342090a0

[3] M. A. Kirchberger, M. Tada and A. M. Katz, "Phospholamban: A Regulatory Protein of the Cardiac Sarcoplasmic Reticulum," Recent Advances in Studies on Cardiac Structure and Metabolism, Vol. 5, 1975, pp. 103115.

[4] J. Fuji, A. Ueno, K. Kitano, S. Tanaka, M. Kadoma and M. Tada, "Complete Complementary DNA-Derived Amino Acid Sequence of Canine Cardiac Phospholamban,” Journal of Clinical Investigation, Vol. 79, No. 1, 1987, pp. 301-304. doi:10.1172/JCI112799

[5] Q. Yao, J. L. Bevan, R. F. Weaver and D. J. Bigelow, "Purification of Porcine Phospholamban Expressed in Escherichia Coli,” Protein Expression and Purification, Vol. 8, No. 4, 1996, pp. 463-468.

[6] J. G. Collins, E. G. Kranias, A. S. Reeves, L. M. Bilezikjian and A. Schwartz, "Isolation of Phospholamban and a Second Proteolipid Component from Canine Cardiac Sar- coplasmic Reticulum,” Biochemical and Biophysical Research Communications, Vol. 99, No. 3, 1981, pp. 796803. doi:10.1016/0006-291X(81)91235-3

[7] E. J. Mayer, E. McKenna, V. M. Garsky, C. J. Burke, H. Mach, C. R. Middaugh, M. Sardana, J. S. Smith and R.G. Johnson Jr., "Biochemical and Biophysical Comparison of Native and Chemically Synthesized Phospholamban and a Monomeric Phospholamban,” Journal of Biologyical Chemistry, Vol. 271, 1996, pp. 1669-1677. doi:10.1074/jbc.271.3.1669

[8] D. J. Hirsh, J. Hammer, W. L. Maloy, J. Blazyk and J. Schaefer, "Secondary Structure and Location of a Magainin Analogue in Synthetic Phospholipid Bilayers," Biochemistry, Vol. 35, No. 39, 1996, pp. 12733-12741. doi:10.1021/bi961468a

[9] A. Mascioni, C. Karim, J. Zamoon, D. D. Thomas and G. Veglia, "Solid-State NMR and Rigid Body Molecular Dynamics to Determine Domain Orientations of Monomeric Phospholamban,” Journal of the American Chemical Society, Vol. 124, No. 32, 2002, pp. 9392-9393. doi:10.1021/ja026507m

[10] E. K. Tiburu, P. C. Dave, K. Damodaran and G. A. Lorigan, "Investigating the Dynamic Properties of the Transmembrane Segment of Phospholamban Incorporated into Phospholipid Bilayers Utilizing ${ }^{2} \mathrm{H}$ and ${ }^{15} \mathrm{~N}$ Solid-State NMR Spectroscopy,” Biochemistry, Vol. 43, No. 44, 2004, pp. 13899-13909. doi:10.1021/bi0490993

[11] E. K. Tiburu, P. C. Dave, J. F. Vanlerberghe, T. B. Cardon, R. E. Minto and G. A. Lorigan, "An Improved Synthetic and Purification Procedure for the Hydrophobic Segment of the Transmembrane Peptide Phospholamban,” Analytical Biochemistry, Vol. 318, No. 1, 2003, pp. 146-151. doi:10.1016/S0003-2697(03)00141-6 